\title{
Wigner Surmise For Domain Systems
}

\author{
Diego Luis González* \\ Gabriel Téllez ${ }^{\dagger}$ \\ Departamento de Física, Universidad de Los Andes
}

A. A. 4976 Bogotá, Colombia.

\begin{abstract}
In random matrix theory, the spacing distribution functions $p^{(n)}(s)$ are well fitted by the Wigner surmise and its generalizations. In this approximation the spacing functions are completely described by the behavior of the exact functions in the limits $s \rightarrow 0$ and $s \rightarrow \infty$. Most non equilibrium systems do not have analytical solutions for the spacing distribution and correlation functions. Because of that, we explore the possibility to use the Wigner surmise approximation in these systems. We found that this approximation provides a first approach to the statistical behavior of complex systems, in particular we use it to find an analytical approximation to the nearest neighbor distribution of the annihilation random walk.
\end{abstract}

Keywords: Systems out of equilibrium, random matrices, Wigner surmise.

\section{Introduction}

In random matrix theory the analytic expressions for the spacing distribution functions of eigenvalues $p^{(n)}(s)$ in the circular and Gaussian orthogonal ensembles (COE and GOE respectively) in the limit of large matrices are

*die-gon1@uniandes.edu.co

†gtellez@uniandes.edu.co 
given in terms of the eigenvalues $\mu_{i}$ and eigenfunctions $f_{i}(x)$ of the following integral equation, see Ref. [1]:

$$
\mu_{i} f_{i}(x)=\int_{-1}^{1} e^{i \pi x y s / 2} f_{i}(y) d y .
$$

The spacing distributions are calculated explicitly by using

$$
\begin{aligned}
& E(2 r, s)=\prod_{i=0}^{\infty}\left(1-\lambda_{2 i}\right) \sum_{0 \leq j_{1}<j_{2}<\cdots<j_{r}} \prod_{i=1}^{r}\left(\frac{\lambda_{j_{i}}}{1-\lambda_{j_{i}}}\right) \times\left[1-\left(b_{j_{1}}+\cdots+b_{j_{r}}\right)\right], \\
& E(2 r-1, s)=\prod_{i=0}^{\infty}\left(1-\lambda_{2 i}\right) \sum_{0 \leq j_{1}<j_{2}<\cdots<j_{r}} \prod_{i=1}^{r}\left(\frac{\lambda_{j_{i}}}{1-\lambda_{j_{i}}}\right) \times\left(b_{j_{1}}+\cdots+b_{j_{r}}\right),
\end{aligned}
$$

where

$$
\begin{gathered}
b_{j}=f_{2 j}(1) \int_{-1}^{1} f_{2 j}(x) d x / \int_{-1}^{1} f_{2 j}^{2}(x) d x \\
\lambda_{j}=s\left|\mu_{j}\right|^{2} / 4
\end{gathered}
$$

and

$$
p^{(n)}(s)=\frac{d^{2}}{d s^{2}} \sum_{j=0}^{n}(n-j+1) E(j, s) .
$$

These expressions are difficult to manage, however in Ref. [2], the authors find an excellent approximation for spacing distributions $p^{(n)}(s)$ from their well-known behavior in the limits $s \rightarrow 0$ and $s \rightarrow \infty$. This approximation is easy to use and provide an excellent fit to the exact distributions. We will use this approximation many times in this paper, because of that, we summarize now its most important aspects.

By definition, $p^{(n)}(s)$ is the probability density that an interval of length $s$ which starts at a level contains exactly $n$ levels and the next, the $n+1$ level, is in $[s, s+d s]$. In the same way, let $F^{(n)}(s)$ be the probability that an interval of length $s$ which starts at a level, contains $n$ levels. By using this definition we can write 


$$
F^{(n)}(s)=\int_{s}^{\infty}\left(p^{(n)}\left(s^{\prime}\right)-p^{(n-1)}\left(s^{\prime}\right)\right) d s^{\prime} .
$$

Additionally, let $r^{(n)}(s)$ be the probability density that an interval $[0, s]$ which starts at a level at $s=0$ is limited by a level on its right side, under the condition that there are exactly $n$ levels in the interval $(0, s)$, i.e., $r^{(n)}(s)$ is the conditional probability

$$
r^{(n)}(s)=\frac{p^{(n)}(s)}{F^{(n)}(s)},
$$

this probability is called level repulsion function. Following Ref. [2], in the limit $s \rightarrow 0$, this equation can be written as

$$
p^{(n)}(s)=r^{(n)}(s) \int_{0}^{s} p^{(n-1)}\left(s^{\prime}\right) d s^{\prime} .
$$

In the GOE ensemble the matrix elements are chosen using a Gaussian distribution, this fact suggest that $p^{(n)}(s)$ decays as Gaussian function. The appropriate function for fit is

$$
p^{(n)}(s)=A_{n} s^{\alpha_{n}} e^{-B_{n} s^{2}},
$$

under the surmise $r^{(n)}(s) \rightarrow s^{n+1}$ with $s \rightarrow 0$. Additionally, the functions $p^{(n)}(s)$ satisfy the normalization conditions

$$
\int_{0}^{\infty} p^{(n)}(s) d s=1
$$

and

$$
\int_{0}^{\infty} s p^{(n)}(s) d s=1 .
$$

By using the surmise for the level repulsion and the normalization conditions, is straightforward to find [2]

$$
\begin{gathered}
A_{n}=2 \frac{B_{n}^{\left(\alpha_{n}+1\right) / 2}}{\Gamma\left(\frac{\alpha_{n}+1}{2}\right)}, \\
B_{n}=\left[\frac{\Gamma\left(\frac{\alpha_{n}}{2}+1\right)}{(n+1) \Gamma\left(\frac{\alpha_{n}+1}{2}\right)}\right]^{2},
\end{gathered}
$$


where

$$
\alpha_{n}=n+\frac{(n+1)(n+2)}{2} .
$$

Then, the approximate spacing distribution functions $p^{(n)}(s)$ are given explicitly by

$$
p^{(n)}(s)=\left[\frac{\Gamma\left(\frac{\alpha_{n}}{2}+1\right)}{(n+1)}\right]^{\alpha_{n}+1} \frac{2 s^{\alpha_{n}}}{\Gamma\left(\frac{\alpha_{n}+1}{2}\right)^{\alpha_{n}+2}} e^{-\left[\frac{\Gamma\left(\frac{\alpha_{n}}{2}+1\right)}{(n+1) \Gamma\left(\frac{\alpha_{n}+1}{2}\right)}\right]^{2} s^{2}} .
$$

The result obtained for $\alpha_{n}$ coincides with the results obtained by using the exact expression for the spacing distribution functions, see Ref. [1]. Notice that the approximate spacing distributions functions are characterized by the level repulsion, normalization condition, scaling condition for the average spacing and Gaussian decay. This approximation is called generalized Wigner surmise and provides a very good approximation for $p^{(n)}(s)$, because it reproduce not only the distributions behavior in the limits $s \rightarrow 0$ and $s \rightarrow \infty$, but also reproduce their global behavior, as we can see in figure 1 . In particular the function with $n=0$ is called Wigner distribution. This fit allow us calculate also the approximate pair correlation distribution $g(r)$. For this purpose we use

$$
g(r)=\sum_{n=0}^{\infty} p^{(n)}(r)
$$

then

$$
g(r)=2 \sum_{n=0}^{\infty}\left[\frac{\Gamma\left(\frac{\alpha_{n}}{2}+1\right)}{(n+1)}\right]^{\alpha_{n}+1} \frac{r^{\alpha_{n}}}{\Gamma\left(\frac{\alpha_{n}+1}{2}\right)^{\alpha_{n}+2}} e^{-\left[\frac{\Gamma\left(\frac{\alpha_{n}}{2}+1\right)}{(n+1) \Gamma\left(\frac{\alpha_{n}+1}{2}\right)}\right]^{2} r^{2}} .
$$

In figure 1 we can see that this is a good approximation for $g(r)$, however, it is not as useful as the Wigner surmise for $p^{(0)}(s)$ because the exact expression for $g(r)$ is well known and easy to use, see Ref. [1].

In Ref. [3] the authors study the statistical behavior of several out of equilibrium domain systems which evolve with formation of domains which grow in time. For intermediate times where the size of the domains is much smaller than the total size $L$ of the system, the domain size distribution exhibit a 
dynamic scaling. The authors studied the statistical properties of these domains in the scaling regime. They found that the statistical behavior of those is similar to the one in random matrices, for example, the nearest neighbor distribution $p^{(0)}(s)$ of several out of equilibrium domain systems is well fitted by the Wigner surmise which also describe closely the distribution $p^{(0)}(s)$ in the case of the circular and Gaussian orthogonal ensembles in random matrix theory (actually this distribution is exact in the case of $2 \times 2$ matrices). However, the next distributions $(n>0)$ for these systems are different from their counterpart in random matrix theory. Another important aspect is the pair correlation function $g(r)$ which, in COE and GOE ensembles and the coalescing random walk and interacting random walk does not have any oscillation but in other systems $g(r)$ describe one oscillation near to $r=1$. For more information see Refs. [3, 4, 5, 6]. In most of the non equilibrium domain systems, the main problem is the absence of analytical expressions for the spacing and correlation functions. Then, the question is: can the generalized Wigner surmise provide a good approximation for $p^{(n)}(s)$ and $g(r)$ in the domain systems as it happens with the random matrix ensembles?

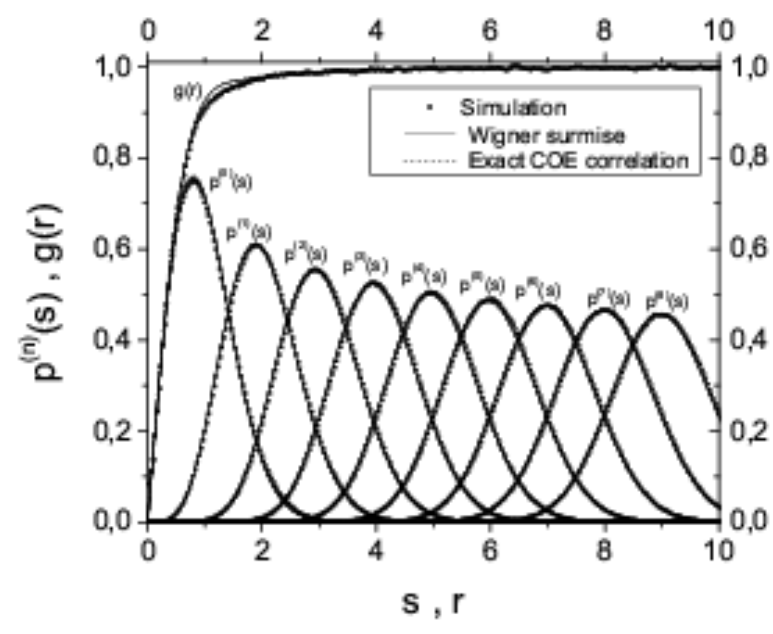

Figure 1: Comparison between the generalized Wigner surmise and the COE ensemble. In the simulation we took 20000 matrices of size $200 \times 200$. 


\section{Wigner surmise for domains systems}

For all systems considered in this paper $p^{(0)}(s)$ is well described by the Wigner distribution, because of that and following the method used in the random matrix theory we propose the next model

$$
\alpha_{n}=\left\{\begin{array}{cc}
1 & \text { for } n=0 \\
h(n) & \text { for } n \geq 1
\end{array}\right.
$$

with $h(n)$ is a function to determine. The spacing distribution functions in this model are given by

$$
p^{(n)}(s)=\left\{\begin{array}{cc}
\frac{\pi}{2} s e^{-\frac{\pi}{4} x^{2}} & \text { if } n=0 \\
A_{n} s^{\alpha_{n}} e^{-B_{n} x^{\beta_{n}}} & \text { if } n \geq 1
\end{array}\right.
$$

using (11) and (12), we find

$$
A_{n}=\frac{\beta_{n} B_{n}^{\frac{1+\alpha_{n}}{\beta_{n}}}}{\Gamma\left(\frac{1+\alpha_{n}}{\beta_{n}}\right)},
$$

and

$$
B_{n}=\left(\frac{\Gamma\left(\frac{2+\alpha_{n}}{\beta_{n}}\right)}{(1+n) \Gamma\left(\frac{1+\alpha_{n}}{\beta_{n}}\right)}\right)^{\beta_{n}} .
$$

\subsection{Independent interval approximation model (IIA)}

The independent intervals are used as an approximate solution in many equilibrium and non equilibrium systems $[3,7,8$, in order to find analytical results. In this approximation, $p^{(n)}(s)$ is given by the convolution product of $n+1$ nearest neighbor distribution factors, because of that, the spacing distribution functions can be calculated by using the Laplace transformation, see Ref. [7]. In particular, in Ref. [3] the IIA is used to find an approximate model for the statistical behavior of two non equilibrium systems which will be explained in next sections.

\subsubsection{Independent interval model for small values of $s$}

In Ref. [3] the authors choose $p^{(0)}(s)$ equal to the Wigner distribution. In order to apply the method of the last section, we need to know the behavior 
of $p^{(n)}(s)$ for small and large values of $s$. For the first region we expand the Wigner distribution in power series

$$
p^{(0)}(s)=\frac{\pi}{2} s e^{-\frac{\pi}{4} s^{2}}=\frac{\pi}{2} s\left(1-\frac{\pi}{4} s^{2}+\cdots\right),
$$

then, to the first order, the nearest neighbor distribution $p^{(0)}(s)$ has a lineal behavior, given by

$$
p^{(0)}(s) \propto s .
$$

In the same limit $s \rightarrow 0$, by using the independent interval approximation for arbitrary values of $n$, we have

$$
p^{(n)}(s) \propto \int_{0<x_{1}<x_{2} \cdots<x_{n}<s} x_{1}\left(x_{2}-x_{1}\right) \cdots\left(s-x_{n}\right) d x_{1} \cdots d x_{n},
$$

which can be evaluated by using the Laplace transform

$$
\widetilde{p}^{(n)}(t) \propto \frac{1}{t^{2(n+1)}},
$$

and then, taking its inverse

$$
p^{(n)}(s) \propto s^{2 n+1} .
$$

As consequence, in the IIA case the exponent $\alpha_{n}$ depends linearly on $n$

$$
\alpha_{n}=2 n+1 \text {. }
$$

By using this result it is possible to determine the behavior of the level repulsion function for $s \rightarrow 0$. Following Ref. [2] we have

$$
r^{(n)}(s) \propto s^{f(n)},
$$

where $f(n)$ is the function to determine. By using equation (9), we can write

$$
p^{(n)}(s) \propto s^{f(n)} \int_{0}^{s} p^{(n-1)}\left(s^{\prime}\right) d s^{\prime}
$$

then

$$
p^{(n)}(s) \propto s^{f(n)+\cdots+f(0)+n} .
$$

By comparing (27) with (31) is straightforward to find

$$
f(n)=1,
$$

for all $n \geq 0$, as a consequence

$$
r^{(n)}(s) \propto s, \quad s \rightarrow 0,
$$

then, the level repulsion does not depend on $n$ as it happens in the COE/GOE case. 


\subsubsection{Independent interval model for large values of $s$}

Now, we need the behavior of $p^{(n)}(s)$ for large values of $s$. The exact expression for $p^{(n)}(s)$ is

$$
p^{(n)}(s)=\int_{0<x_{1}<x_{2} \cdots<x_{n}<s} p^{(0)}\left(x_{1}\right) p^{(0)}\left(x_{2}-x_{1}\right) \cdots p^{(0)}\left(s-x_{n}\right) d x_{1} \cdots d x_{n} .
$$

In our case $p^{(0)}(s)$ is given by the Wigner surmise, then

$$
p^{(n)}(s)=\left(\frac{\pi}{2}\right)^{n+1} \int_{0<x_{1}<x_{2} \cdots<x_{n}<s} x_{1} e^{-\frac{\pi}{4} x_{1}^{2}} \cdots\left(s-x_{n}\right) e^{-\frac{\pi}{4}\left(s-x_{n}\right)^{2}} d x_{1} \cdots d x_{n} .
$$

We can calculate the behavior of these functions for arbitrary values of $n$ in this limit $s \rightarrow \infty$ as we show next. From Ref. [3] we know that at least the first two spacing distribution functions decay like Gaussian functions, then, we assume that for arbitrary values of $n$ these functions have the form $p_{a s y}^{(n)}(s)=M_{n} s^{\gamma_{n}} e^{-N_{n} s^{2}}$ in the limit $s \rightarrow \infty$. In order to eliminate the integrals in equation (35) we use the Laplace transformation

$$
\widetilde{p}^{(n)}(l)=\left(1-l e^{l^{2} / \pi} \operatorname{erfc}\left(\frac{l}{\sqrt{\pi}}\right)\right)^{n+1},
$$

where $\operatorname{erfc}(z)=(2 / \sqrt{\pi}) \int_{z}^{\infty} e^{-t^{2}} d t$ is the complementary Gaussian error function. In the same way we take the Laplace transform in $p_{a s y}^{(n)}$. Additionally, we expand both transformations in Taylor series around $l=0$. Let be $Z_{j}$ the $j^{\text {th }}$ coefficient in the expansion of equation (36) and $Y_{j}$ is the one for the Laplace transform of $p_{a s y}^{(n)}(s)$. We find that the coefficients of both expansions satisfy the relation $Y_{i} / Z_{i}=Y_{j} / Z_{j}$ in the limit $i, j \rightarrow \infty$. By using this method we can find $M_{n}, N_{n}$ and $\beta_{n}$. If fact we find that $N_{n}=\frac{\pi}{4 n}$ and $\gamma_{n}=n+1$. In general, if we know $p^{(0)}(s)$ we can calculate the asymptotic behavior of $p^{(n)}(s)$ under the assumption that the IIA is valid for $s \rightarrow \infty$, but, as we will see in next sections, this is not true always.

In the figure 2 we compare the exact statistical behavior of IIA with the generalized Wigner surmise, i.e., with a fit developed by using the behavior of $p^{(n)}(s)$ in the limits $s \rightarrow 0$ and $s \rightarrow \infty$, because of that from now on we will call it local fit. Also, we compare the global fit which was developed by using equations (19) to (22) and the complete behavior of $p^{(n)}(s)$ in the interval 
$[0, \infty]$. By using the values of $\alpha_{n}$ found in the global fit, we developed a new fit to determine the global behavior of $\alpha_{n}$, explicitly in this case we have

$$
\alpha_{n}=1.8268 n+0.9954,
$$

this result is close to the exact exponent (28), even when we use wrong functions in the fit; for example, the exact result for $p^{(1)}(s)$ is, see Ref. [3]

$$
p^{(1)}(s)=\frac{\pi}{16} e^{-\frac{\pi s^{2}}{4}}\left(4 s+\sqrt{2} e^{\frac{\pi s^{2}}{8}}\left(-4+\pi s^{2}\right) \operatorname{erf}\left(\frac{1}{2} \sqrt{\frac{\pi}{2}} s\right)\right),
$$

which is very different form our surmise, however, both functions (20) and (38) have the same type of behavior in the limits $s \rightarrow 0$ and $s \rightarrow \infty$. Equation (18) for the correlation function it is still valid in both cases, global and local fit, we only must use equation (28) and (37) respectively. The main problem in the global fit approximation it is the use of not integer exponents in the level repulsion. Figure 3 show the differences between the three cases for small values of $s$, naturally in this region the graph of the global fit is not parallel to graph of the exact result as it actually happens in the local fit approximation. In figure 4 we can see the linear behavior of $p^{(n)}(s)$ in limit $s \rightarrow \infty$, which implies that the distribution functions decay like a Gaussian function as it was to be expected.

\subsection{Coalescing random walk (CRW)}

In the coalescing random walk the particles describe independent random walks along a one dimensional lattice and they are subjected to the reaction $A+A \rightarrow A$. This system is well studied [4, 9, 10, 11] and its analytical solution is well know, because of that is used as approximation to more complex systems. Let $q^{(n)}(s)$ be the conditional probability that given one particle its next neighbor is at a distance of $s$. From its definition $q^{(n)}(s)$ is given by

$$
q^{(n)}(s)=\int_{0<y_{1}, \cdots,<y_{n}<s} \omega^{(n+2)}\left(y_{1}, \cdots, y_{n}, s\right) d y_{1}, \cdots, d y_{n}
$$

with

$$
\omega^{(n)}\left(x_{1}, \cdots, x_{n}\right)=-\left.\frac{\partial^{n} E^{(n-1)}\left(x_{1}, y_{1}, \cdots, x_{n-1}, y_{n-1}\right)}{\partial x_{1} \cdots \partial x_{n-1} \partial y_{n-1}}\right|_{y_{1}=x_{2}, \cdots, y_{n-1}=x_{n}},
$$




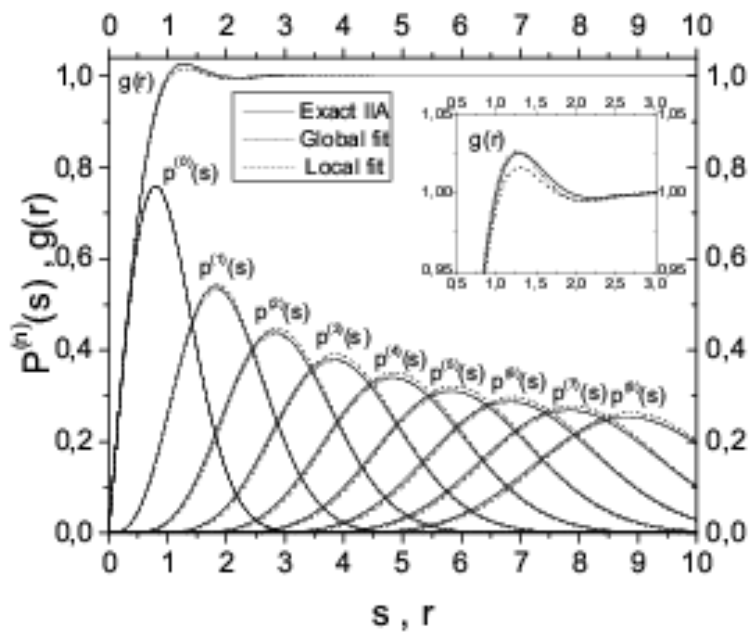

Figure 2: Comparison between the exact statistical behavior of IIA, the generalized Wigner surmise (local fit) and the global fit.

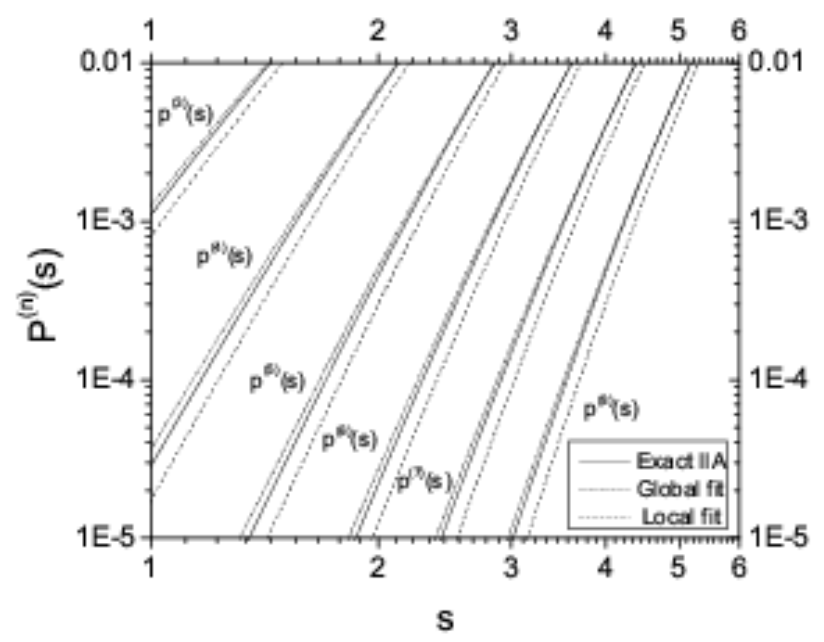

Figure 3: Log-Log graphic for the spacing distribution functions for IIA. 


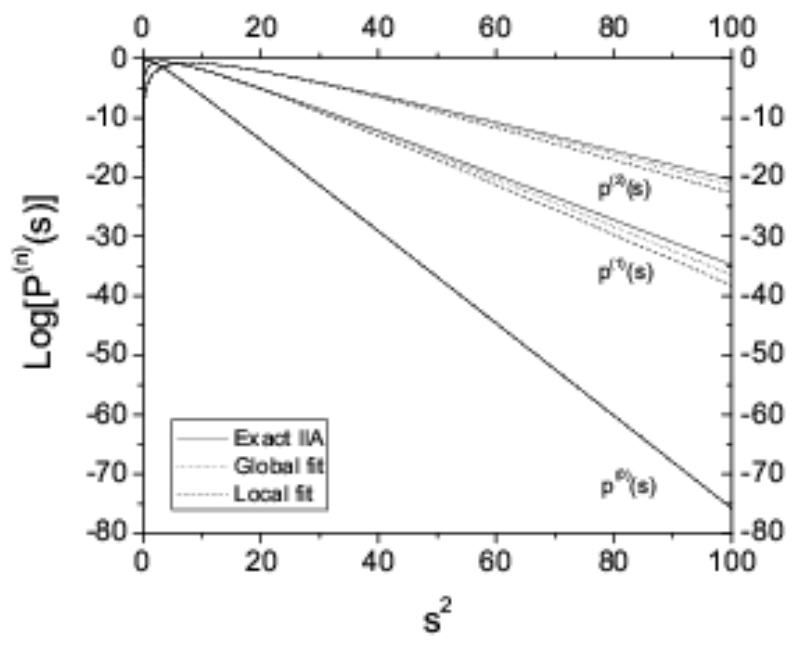

Figure 4: Asymptotic behavior for $s \rightarrow \infty$ of the spacing distribution functions.

$$
E^{(n)}\left(x_{1}, y_{1}, \cdots, x_{n}, y_{n}, t\right)=\sum_{p=1}^{(2 n-1) ! !} \sigma_{p} E^{(1)}\left(z_{1, p}, z_{2, p}, t\right) \cdots E^{(1)}\left(z_{2 n-1, p}, z_{2 n, p}, t\right)
$$

where $z_{1, p}, z_{2, p}, \ldots, z_{2 n, p}$ symbolize an ordered permutation, $p$, of the variables $x_{1}, y_{1}, \ldots, x_{n}, y_{n}$, such that

$$
z_{1, p}<z_{2, p}, z_{3, p}<z_{4, p}, \cdots, z_{2 n-1, p}<z_{2 n, p}
$$

and

$$
z_{1, p}<z_{3, p}<z_{5, p} \cdots<z_{2 n-1, p}
$$

The function $E^{(1)}\left(x_{1}, y_{1}, t\right)$ is the probability that from $x_{1}$ to $y_{1}$ the lattice is empty at time $t$. Then it is possible generate the complete solution for the CRW from $E^{(1)}\left(x_{1}, y_{1}, t\right)$, which is given by the solution of the diffusion equation under the suitable boundary conditions (see Ref. [4]). In fact, the 
exact expression for this function is

$$
E^{(1)}\left(x_{1}, y_{1}, t\right)=\operatorname{erfc}\left(\frac{y_{1}-x_{1}}{\sqrt{8 D t}}\right)
$$

with $D$ the diffusion constant and $t$ the time, for additionally information see Ref. 4]. For practical purposes, the solution given by equations (39) to (44) is hard to evaluate for arbitrary values of $n$ but it can be evaluated in the limit $s \rightarrow 0$ using Taylor series. The case $n=0$ is trivial, the Taylor expansion for equation (44) is

$E^{(1)}\left(x_{1}, y_{1}, t\right)=1-\frac{y_{1}-x_{1}}{\sqrt{2 \pi}(D t)^{1 / 2}}+\frac{\left(y_{1}-x_{1}\right)^{3}}{24 \sqrt{2 \pi}(D t)^{3 / 2}}-\frac{\left(y_{1}-x_{1}\right)^{5}}{640 \sqrt{2 \pi}(D t)^{5 / 2}}+O(x, y)^{7}$

then

$$
\begin{gathered}
q^{(0)}\left(x_{2}, x_{1}\right)=\omega^{(2)}\left(x_{1}, x_{2}\right)=-\left.\frac{\partial^{2}}{\partial x_{1} \partial y_{1}} E^{(1)}\left(x_{1}, y_{1}, t\right)\right|_{y_{1}=x_{2}}, \\
q^{(0)}\left(x_{2}, x_{1}\right)=\frac{x_{2}-x_{1}}{4 \sqrt{2 \pi}(D t)^{3 / 2}}-\frac{\left(x_{2}-x_{1}\right)^{3}}{32 \sqrt{2 \pi}(D t)^{5 / 2}}+O(x)^{5} .
\end{gathered}
$$

Making the variable change $s=\frac{x_{2}-x_{1}}{\sqrt{2 \pi D t}}$ and taking into account that $p^{(0)}(s)=$ $2 \pi D t q^{(0)}\left(x_{2}, x_{1}\right)$, the product $D t$ disappears (dynamical scaling) in the above equation. Then, to first order, we have

$$
p^{(0)}(s)=\frac{s \pi}{2}+O(s)^{3} .
$$

For small values of $s, p^{(0)}(s)$ has a linear behavior, i.e., $\alpha_{0}=1$. The case $n=1$ is more complicated, in fact we have

$$
\omega^{(3)}\left(x_{1}, x_{2}, x_{3}\right)=-\left.\frac{\partial^{3}}{\partial x_{1} \partial x_{2} \partial y_{2}} E^{(2)}\left(x_{1}, y_{1}, x_{2}, y_{2}, t\right)\right|_{y_{1}=x_{2}, y_{2}=x_{3}}
$$

where

$$
\begin{aligned}
E^{(2)}\left(x_{1}, y_{1}, x_{2}, y_{2}, t\right) & =E\left(x_{1}, y_{1}, t\right) E\left(x_{2}, y_{2}, t\right) \\
& +E\left(x_{1}, y_{2}, t\right) E\left(y_{1}, x_{2}, t\right) \\
& -E\left(x_{1}, x_{2}, t\right) E\left(y_{1}, y_{2}, t\right)
\end{aligned}
$$


then

$$
\omega^{(3)}\left(x_{1}, x_{2}, x_{3}\right)=\frac{\left(x_{2}-x_{1}\right)\left(x_{3}-x_{1}\right)\left(x_{3}-x_{2}\right)}{32 \pi(D t)^{3}}+O(x)^{4},
$$

in that way $q^{(1)}\left(x_{1}, x_{3}, t\right)$ is given by

$$
q^{(1)}\left(x_{3}, x_{1}, t\right)=\int_{x_{1}}^{x_{3}} \frac{\left(x_{2}-x_{1}\right)\left(x_{3}-x_{1}\right)\left(x_{3}-x_{2}\right)}{32 \pi(D t)^{3}} d x_{2}+O(x)^{5} .
$$

Integrating

$$
q^{(1)}\left(x_{3}, x_{1}, t\right)=\frac{\left(x_{3}-x_{1}\right)^{4}}{192 \pi(D t)^{3}}+O(x)^{5} .
$$

Using again the variable change, it is straigthfoward to find

$$
p^{(1)}(s)=\frac{\pi^{2} s^{4}}{24}+O(x)^{5}
$$

we conclude that $\alpha_{1}=4$. In general for an arbitrary value of $n$, we find that the first term in the expansion is

$$
q^{(n)}\left(x_{1}, x_{n}, t\right) \propto \int_{x_{1}}^{x_{n}} \cdots \int_{x_{1}}^{x_{3}} \prod_{1 \leq i<j \leq n}\left(x_{j}-x_{i}\right) d x_{2} \cdots d x_{n-1}
$$

therefore, the above equation has $(n+1)(n+2) / 2$ different factors which implies that the integrand is proportional to $x_{i}^{(n+1)(n+2) / 2}$. Making the integral and the usual variable change, the final expression for small values of $s$ is proportional to $s^{(n+1)(n+2) / 2+n}$, explicitly, we have

$$
\alpha_{n}=n+\frac{(n+1)(n+2)}{2} \text {. }
$$

This is the same result reported in Ref. [2] for the GOE/COE case and coincides with the partial result presented in Ref. [11] for the CRW. We made again both fits, global and local. The global fit was made with the data from our simulation where we use a lattice with 1000 sites and 500 particles in $t=0$. The data to build the histograms was taken at three different times $T=50, T=100$ and $T=200$ over 50000 realizations. In this case the global fit is not as accurate as in the IIA case as we can see in figure 5 but it still is a good approximation. We use again equations (19) to (22); and additionally we supposed a Gaussian decay $(\beta=2)$. The global fit gives 


$$
\alpha_{n}=2.8688 n+0.8621 .
$$

The global fit gives an erroneous exponent which depend linearly with $n$, this result it does not coincide with the analytical result (56), where, $\alpha_{n}$ is a quadratic function of $n$. The local fit it is very different from the simulation results and coincides with the statistical behavior of the $\mathrm{COE} / \mathrm{GOE}$ ensembles.

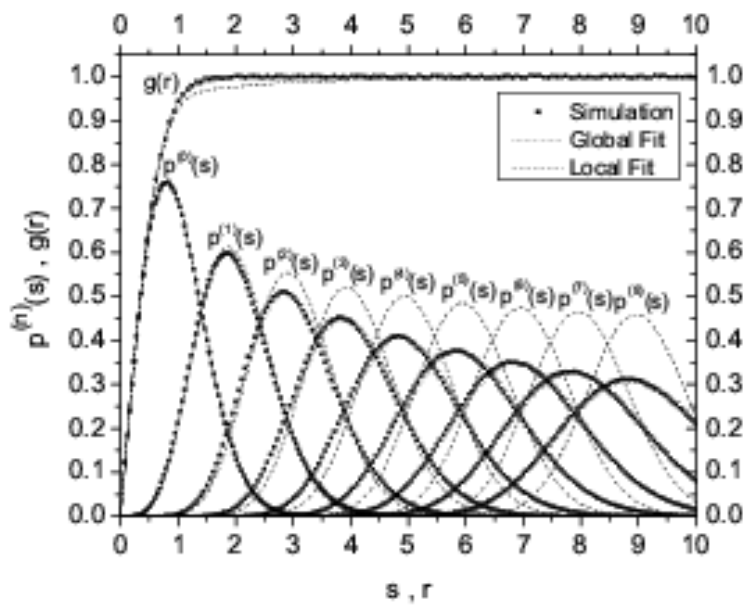

Figure 5: Comparison between the statistical behavior of CRW, the global fit and the local fit.

Although the global and local fit models are approximate, we can use them as a good approximations in some cases. For example, in Ref. 11 the authors find an exact relation for the nearest neighbor distribution $p_{a n n}^{(0)}(s)$ in the annihilation random walk in terms of $p^{(n)}(s)$ of the coalescing random walk. Explicitly, they found

$$
p_{\text {ann }}^{(0)}(s)=\sum_{n \geq 0} \frac{1}{2^{n}} p^{(n)}(2 s),
$$

In order to test the validity of our approximations, we implement a simulation for the annihilation random walk for a one dimensional lattice with 


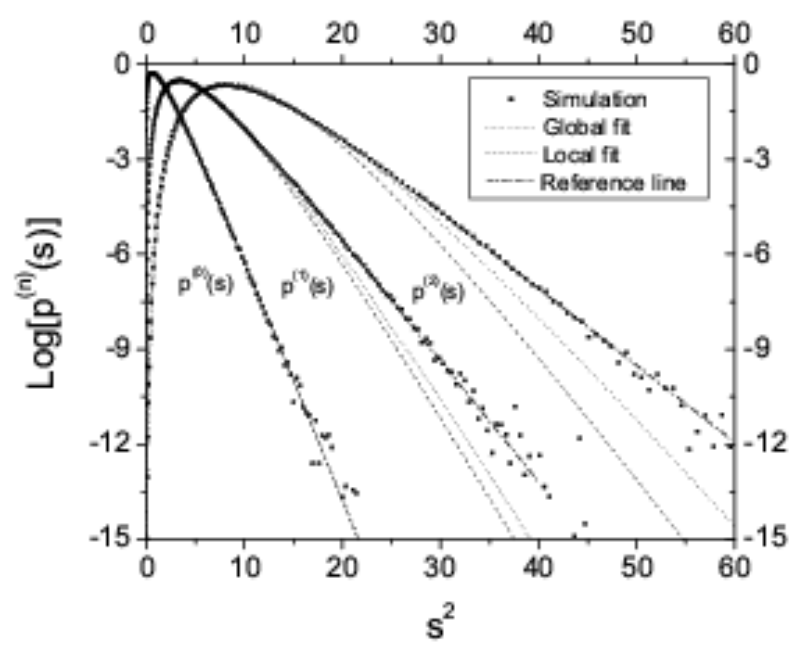

Figure 6: Log-Log graphic for the spacing distribution functions of the CRW.

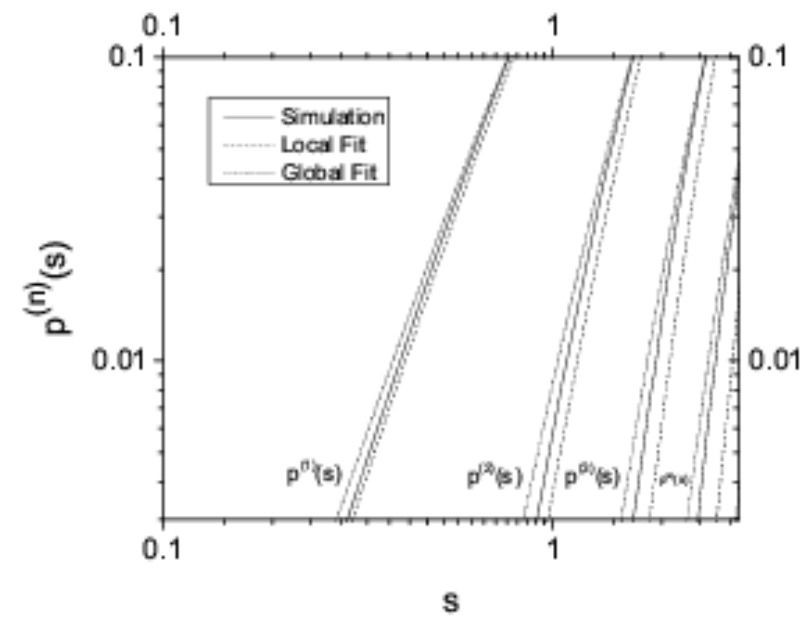

Figure 7: Statistical behavior of CRW for small values of $s$. 
2000 sites, 100 particles at $t=0$ over 20000 realizations, the histogram was build by using three times $T=1000, T=1500$ and $T=2000$. By using the global and the local fit for the distribution functions $p^{(n)}(s)$ of the CRW, with equation (58), we find two analytical models for the annihilation random walk. We can see in figure 8 that the global and local fits provides a good approximation for the nearest neighbor distribution of the annihilation random walk. Additionally, figure 9 compare global and local fit with the asymptotic result $p_{a n n}^{(0)}(s) \approx 1.8167 e^{-1.3062 s}$ given in Ref. [11.

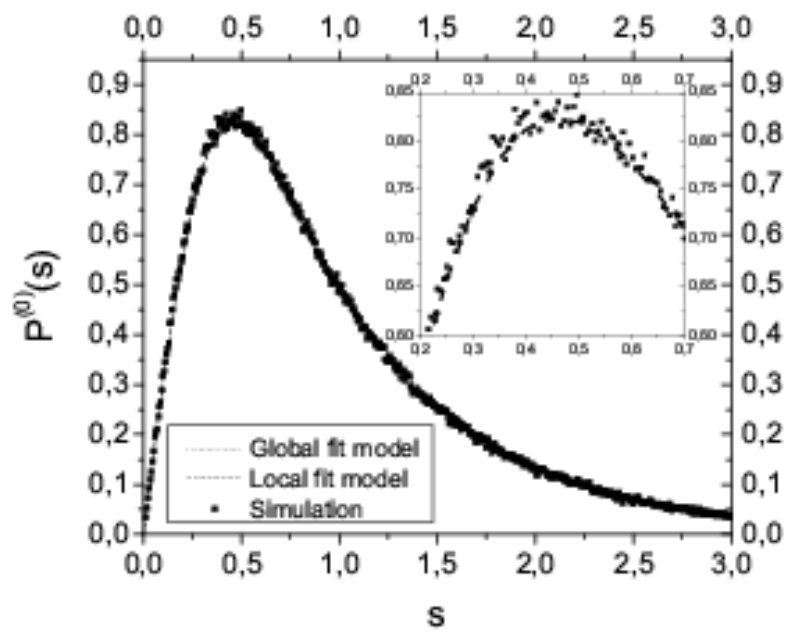

Figure 8: Approximation for $p_{a n n}^{(0)}(s)$ by using global and local fits.

\subsection{Spin System}

This system was introduced in Ref. [5], where the authors consider a chain of $L$ Ising spins with nearest neighbor ferromagnetic interaction $J$. The chain is subject to spin-exchange dynamics with a driving force $E$ that favors motion of up spins to the right over motion to the left. In this case we do not have an analytical solution for the spacing distribution functions, because of that, we must start exploring numerically the behavior of $p^{(n)}(s)$ for small and large values of $s$. In figure 10, we can see the linear behavior of the 


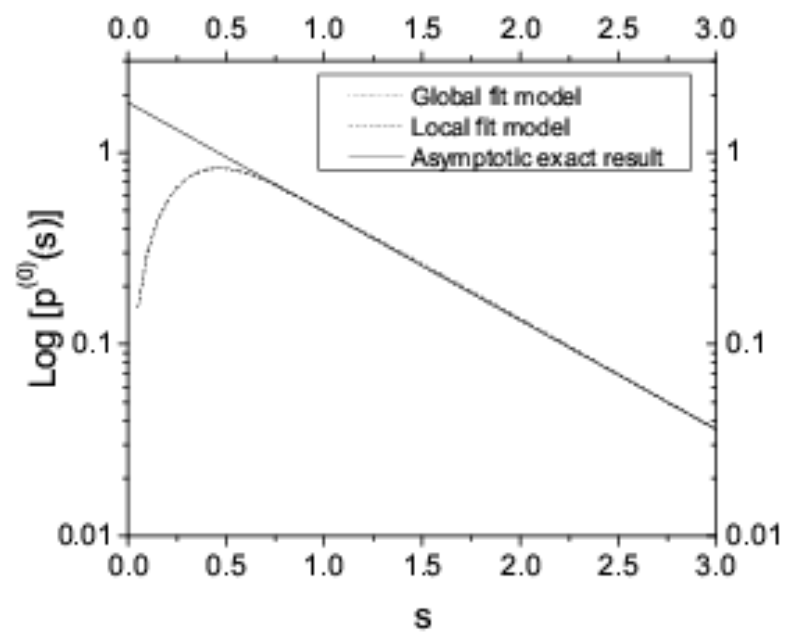

Figure 9: Asymptotic behavior of $p_{a n n}^{(0)}(s)$.

spacing distribution function for $s \rightarrow 0$. Using values in this region we develop a fit which suggest that $\alpha_{1}=3$ and $\alpha_{2}=6$ approximately. Naturally $\alpha_{0}=1$, however it is very difficult to know using this method the next exponents because it is not possible develop a numerical simulation with enough precision.

Curiously, these exponents for $n=0,1,2$ are given by the equation

$$
\alpha_{n}=\frac{(n+1)(n+2)}{2}
$$

which is very similar to its counterpart in COE and GOE cases. For $s \rightarrow \infty$, $p^{(n)}(s)$ decay like a Gaussian function as we can see in figure 11, In this case the global fit gives

$$
\alpha_{n}=1.270 n+0.920 .
$$

In figure 12, we show the results given by equations (19) to (22) for the global fit in comparison with the simulation results which was made with a lattice with 1000 sites, equal number of spins up and down taken at two times $t=34$ and $t=48$ to build the histograms. The result for $g(r)$ is very good 


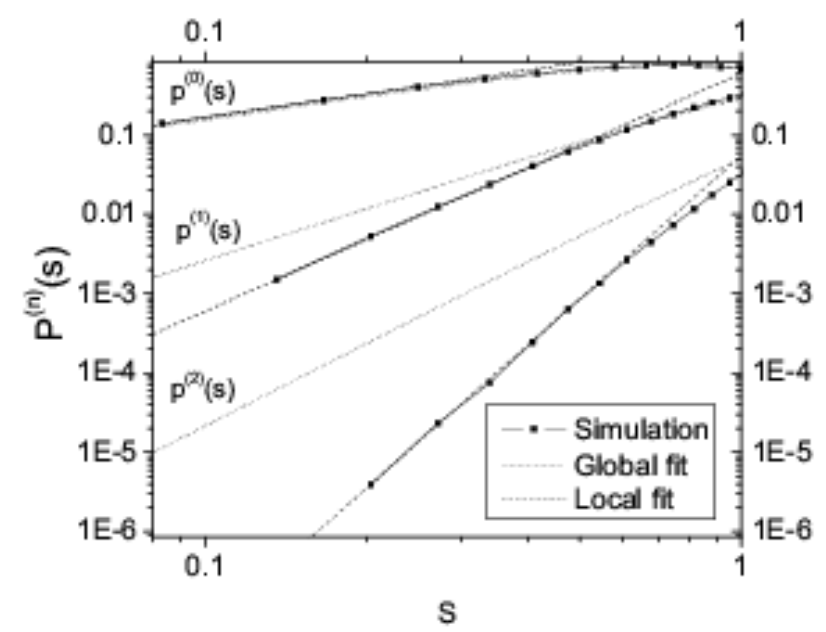

Figure 10: Log-Log graphic for the spacing distribution functions of spin system.

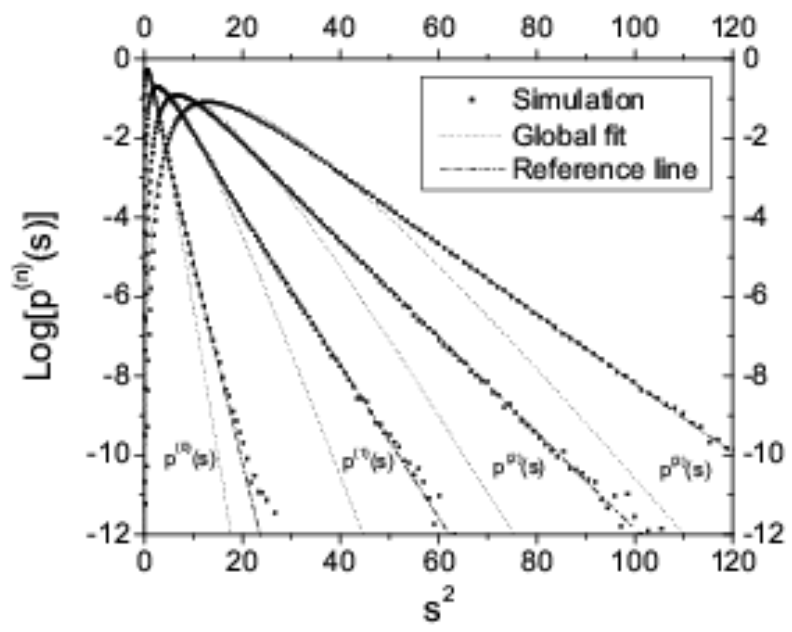

Figure 11: Asymptotic behavior of the spin system. 
with a maximum error of $2.5 \%$. Unfortunately this approximation is not good enough for $p^{(n)}(s)$ but at least it reproduce qualitatively the behavior of the real functions for $s \rightarrow \infty$. The local fit gives terrible results as it happens in the CRW case.

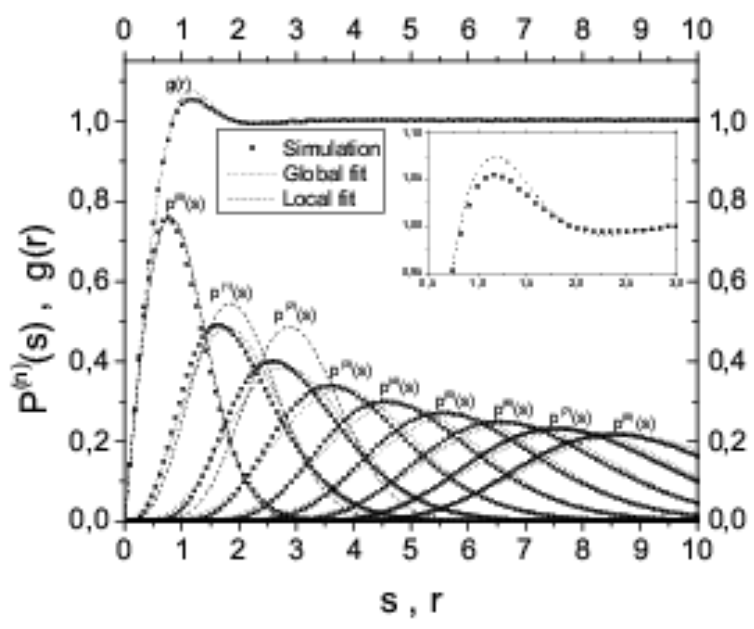

Figure 12: Comparison between the statistical behavior of the spin system and the global fit.

\subsection{Gas System}

This system was originally studied in [6]. There, the authors studied the biased diffusion of two species in a fully periodic $2 \times L$ rectangular lattice half filled with two equal number of two types of particles (labeled by their charge + or -$)$. An infinite external field drives the two species in opposite directions along the $x$ axis (long axis). The only interaction between particles is an excluded volume constraint, i.e., each lattice site can be occupied at most by only one particle. As it happens in the spin system, we do not know an analytic solution for the spacing and pair correlation functions. We follow the same method used in the spin system. In figure 13, we can see the linear behavior of $p^{(n)}(s)$ which, by fit, give us $\alpha_{1}=3$ and $\alpha_{2}=5$ approximately, 
and of course $\alpha_{0}=1$. This fact suggest a linear behavior for $\alpha_{n}$ given by

$$
\alpha_{n}=2 n+1
$$

but again we could not find the next exponents with enough precision in order to validate the above equation. For $s \rightarrow \infty$ we found that $p^{(0)}(s)$ decays like a Gaussian function $(\beta=2)$, but for $n>0$, we found that $\beta$ is an indeterminate function of $n$. For example in figure 14 we can see the asymptotic behavior for two consecutive spacing distribution functions, the figure suggest $\beta=2$ for $p^{(0)}(s)$ and $\beta \neq 2$ for $p^{(1)}(s)$ as it happens in Ref. [12]. Because it is difficult determine the exact value of $\beta$ from the graphics, we implement a linear regression to find which value of $\beta$ give us a better "straight" line. With this method we find for example, that $\beta=2.6$ for $n=1, \beta=3$ for $n=5$ and $\beta=3.2$ for $n=8$. In the linear regressions we took values between $5.5 \geq s \geq 2.5,11.7 \geq s \geq 7$ and $15.5 \geq s \geq 10$ respectively. Because of that, for the gas system we propose a model where $\beta$ depends on $n$. In particular we choose $\beta=2.6+0.1(n-1)$. With this model, the global fit gives

$$
\alpha_{n}=1.016 n+0.788 .
$$

The results of the global fit are show in figure 15, again we find good fit for $g(r)$ with a maximum error of $2 \%$ approximately but the agreement for

$p^{(n)}(s)$ is not so good. Additionally, we include the first spacing distribution obtained with the local fit and our model for $\beta_{n}$.

\section{Conclusion}

In $\mathrm{COE}$ and GOE ensembles, the spacing distribution functions $p^{(n)}(s)$ can be well described by using their behavior for small and large values of $s$ (local fit) as it happens in IIA case, however, this is not true for more complex systems like CRW, spin and gas systems. This result was to be expected because in general the spacing distribution functions are characterized also by their inter medium behavior. In general, the global fit gives better results in comparison with the local fit but it fails to reproduce the level repulsion, in fact, gives non integer exponents. The level repulsion for the CRW has the same behavior that the circular and Gaussian orthogonal ensembles, i.e., both systems are equivalents for $s \rightarrow 0$. The numerical results suggest that 


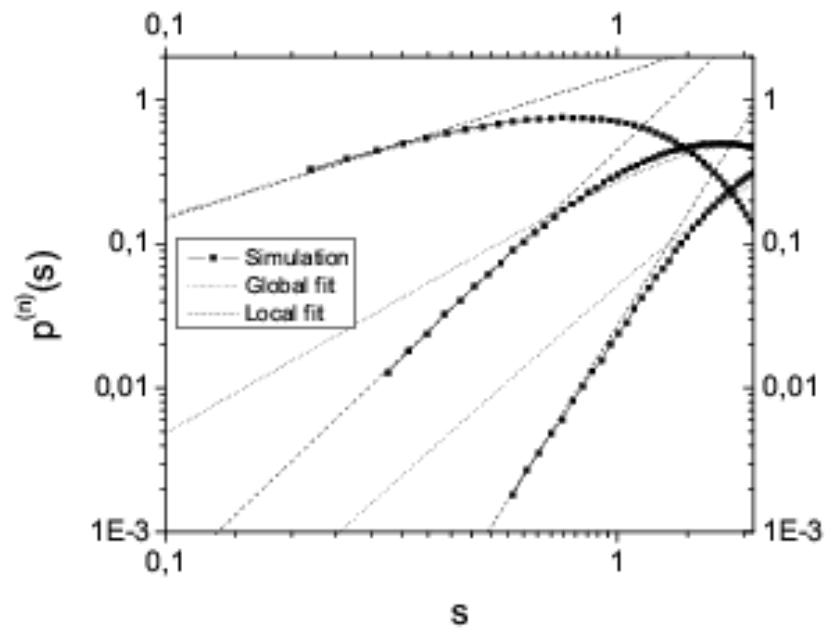

Figure 13: Log-Log graphic for the spacing distribution functions of gas system.

the IIA and the gas system are also equivalents in that region. We find numerical evidence that the spacing distributions functions for gas system is described by a non universal function, in fact, they decay as $M_{n} s^{\gamma_{n}} e^{-N_{n}} s^{\beta_{n}}$ for $n>0$, with $\beta_{n}$ an indeterminate function of $n$. In general the global and local fit provides a first approximation for $p^{(n)}(s)$ and $g(r)$, which can be used as a good approximation as it happens in the annihilation random walk case. These approximations also serve to classify the spacing distribution functions according to their level of repulsion and their decay functional form.

\section{Acknowledgments}

This work was partially supported by an ECOS Nord/COLCIENCIAS action of French and Colombian cooperation and by the Faculty of Sciences of Los Andes University. 


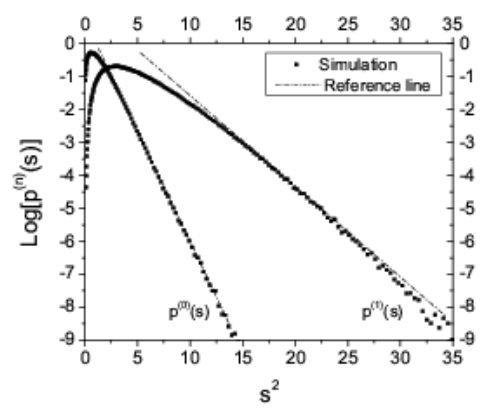

(a)

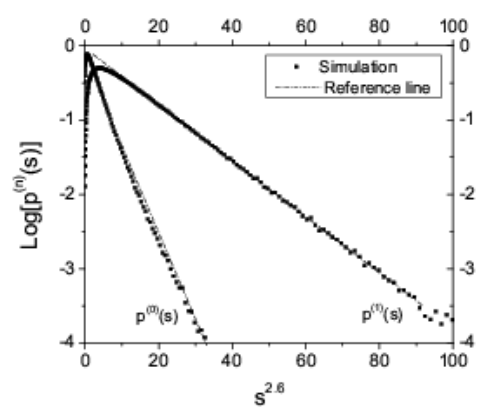

(c)

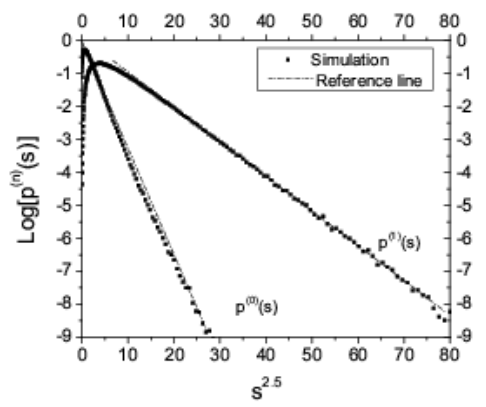

(b)

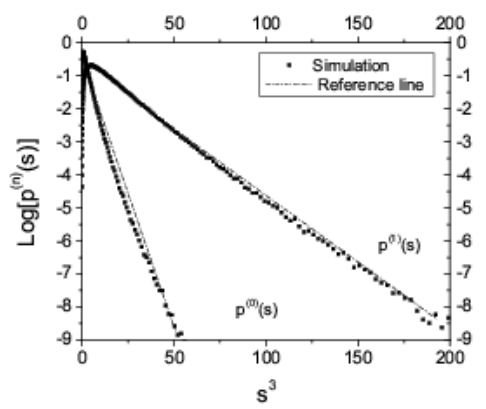

$(d)$

Figure 14: Asymptotic behavior of $p^{(0)}(s)$ and $p^{(1)}(s)$ for the gas system. 


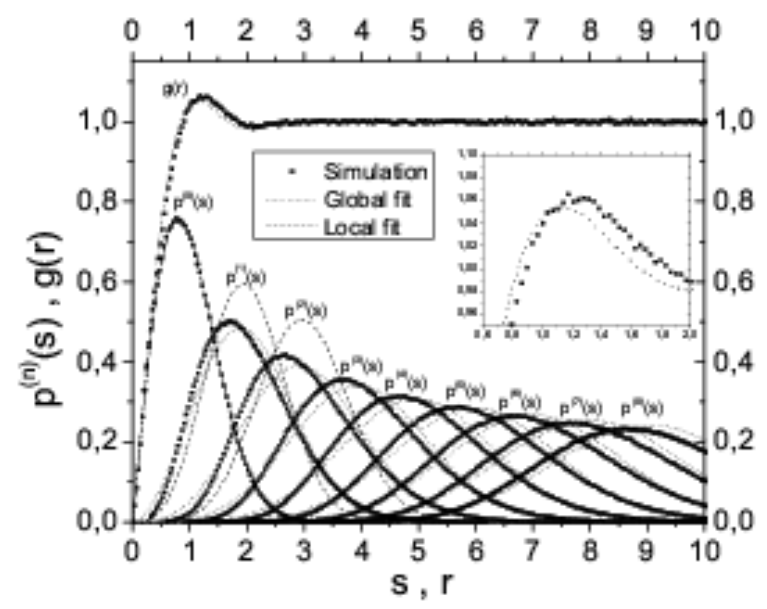

Figure 15: Comparison between the statistical behavior of the gas system and the global fit.

\section{References}

[1] M. Mehta, Random matrices $2^{e d}$, Academic press (1991).

[2] A. Y. Abdul-Magd and M. H. Simbel, Wigner surmise for high-order level spacing distribution of chaotic systems, Phys. Rev E. 60:5371-5374 (1999).

[3] D. L. González and G. Téllez, Statistical behavior of domain systems, Phys. Rev. E. 76:011126 (2007).

[4] D. ben-Avraham and S. Havlin. Diffusion and reactions in fractals and disordered systems, Cambridge University Press (2000).

[5] S. J. Cornell and A. J. Bray, Domain growth in a one-dimensional driven diffusive system, Phys. Rev. E 54:1153-1160 (1996).

[6] J. Mettetal, B. Schmittmann and R. Zia, Coarsening dynamics of a quasi one-dimensional driven lattice gas, Europhysics Lett. 58:653-659 (2002). 
[7] Z. W. Salsburg, R. W. Zwanzig and J. G. Kirkwood, Molecular distribution functions in a one-dimensional fluid, J. Chem. Phys. 21:1098-1107 (1953). Lett. 58:653-659 (2002).

[8] P. A. Alemany and D. ben-Avraham, Inter-particle distribution functions for one-species diffusion-limited annihilation, $A+A \rightarrow 0$, Phys. Lett. A. 206:18-25 (1995).

[9] C. Doering. Physica A, Microscopic spatial correlations induced by external noise in a reaction-diffusion system, 188:386-403 (1992).

[10] D. ben-Avraham, Complete exact solution of diffusion-limited coalescence, $A+A \rightarrow A$, Phys. Rev. Lett. 81:4756-4759 (1998).

[11] D. ben-Avraham and É. Brunet, On the relation between one-species diffusion-limited coalescence and annihilation in one dimension. J. Phys. A: Math. Gen. 38:3247-3252 (2005).

[12] F. D. A. Aarão and R. B. Stinchcombe, Non universal coarsening and universal distributions in far-from-equilibrium systems. Physical Rev. E 71:026110 (2005). 